\title{
Beschreibung des Wachstums weiblicher Rinder verschiedener Rassen
}

\begin{abstract}
Summary
Title of the paper: Description on the growth of female cows of different race

Continuously won weight data from the birth up to the adult animal of female cows of the races Charolais, Fleckvieh and Uckermarker were used for the determination of best fitted functions with respect to some optimum functions from different growth-functions like exponential-, Gompertz-, logistic-, Bertalanffy-, tanhand Janoschek-function. The four-parametric Richards- and Janoschek-function describe the natural growth (monthly increases) most exact. The exponential-function is also suitable for the describtion of the weightdevelopment.
\end{abstract}

Key words: growth, beef cattle, breeds, function, optimum

\section{Zusammenfassung}

Kontinuierlich gewonnene Wägedaten von der Geburt bis zum erwachsenen Tier von weiblichen Fleischrindern der Rassen Charolais, Fleckvieh und Uckermärker wurden dazu genutzt, aus verschiedenen Wachstumsfunktionen wie Exponential-, Gompertz-, Logistische -, Bertalanffy-, tanh-, Janoschek- und Richards-Funktion diejenigen zu bestimmen, die beste Anpassungen im Sinne bestimmter Optimalitätskriterien liefern. Die vierparametrigen Richards- und Janoschek-Funktionen beschreiben den natürlichen Wachstumsverlauf (Zunahme/Monat) am genausten. Für den Verlauf der Gewichtsentwicklung ist daneben auch die Exponentialfunktion geeignet.

Schlïsselwörter: Wachstum, Fleischrind, Funktion, Optimalităt

1. Einleitung

Aufgrund von Meßwerten der Lebendmasse verschiedener Rinderrassen zu unterschiedlichen Lebensaltern sollen aus einer Reihe geeigneter Wachstumsfunktionen diejenigen bestimmt werden, die das Wachstum im Sinne bestimmter Optimalitätskriterien am besten beschreiben. Als Optimalitätskriterien werden einmal Modifikationen eines von MATTHES et al. (1996) vorgeschlagenen Optimalitätskriteriums verwendet. Diese Kriterien berücksichtigen neben der geschätzten Restvarianz im entsprechenden nichtlinearen Regressionsmodell auch, wie durch die jeweilige Funktion die Geburtsund Lebendendmassen geschätzt werden. Außerdem werden auch Anpassungskriterien diskutiert, die die unterschiedliche Parameteranzahl der verwendeten Wachstumsfunktionen berücksichtigen. 
2 .

2.1

Material und Methode

Das verwendete Datenmaterial, das bis zum Jahr 1991 an Rinderbeständen in zwei Betrieben in Mecklenburg-Vorpommern gewonnen wurde, enthält Messungen der Körpermasse (in kg) männlicher und weiblicher Rinder der Rassen Charolais, Fleckvieh und Uckermärker für unterschiedlichste Lebensalter (in Monaten). Die Rinder befanden sich von Anfang Mai bis Ende Oktober auf der Weide und wurden im Winter im Stall mit Silage und Heu gefüttert. Nach der Abkalbung erhielten die Mutterkühe bis zu $3 \mathrm{~kg}$ Konzentrat pro Tag. Auf der Weide wurde nicht zugefüttert. Mineralstoffe wurden das ganze Jahr über verabreicht.

In den nach Rasse, Geschlecht und Betrieb klassifizierten Daten schwankt die Anzahl der Beobachtungen in den besetzten Klassen zwischen 4 und 12327. Die genaue Anzahl der Beobachtungen (N) sowie die jeweils kleinsten und größten Werte von Alter und Lebendmasse (LM) in den einzelnen Klassen ist aus Tabelle 1 ersichtlich.

Tabelle 1

Minimum und Maximum von Alter und Lebendmasse der Meßwerte (Minimum and maximum of age and weight of the observations)

\begin{tabular}{|c|c|c|c|c|c|c|c|}
\hline Rasse & Geschl. & Betrieb & $\mathrm{N}$ & min. Ảlter & $\max$. Alter & min. LM & $\max . \mathrm{LM}$ \\
\hline \multirow[t]{8}{*}{ Charolais } & 1 & 1 & 197 & 0 & 12,98 & 34 & 580 \\
\hline & & 2 & 5361 & 0 & 13,05 & 23 & 633 \\
\hline & 2 & 1 & 545 & 0 & 72,70 & 24 & 760 \\
\hline & & 2 & 6014 & 0 & 74,50 & 22 & 834 \\
\hline & 3 & 1 & . & . & . & . & . \\
\hline & & 2 & 42 & 0 & 56,30 & 23 & 658 \\
\hline & 4 & 1 & 4 & 0 & 6,50 & 27 & 217 \\
\hline & & 2 & 12 & 0 & 7,40 & 25 & 279 \\
\hline \multirow[t]{8}{*}{ Fleckvieh } & 1 & 1 & 137 & 0 & 12,95 & 28 & 549 \\
\hline & & 2 & 442 & 0 & 11,97 & 23 & 500 \\
\hline & 2 & 1 & 972 & 0 & 95,50 & 29 & 700 \\
\hline & & 2 & 197 & 0 & 67,70 & 25 & 760 \\
\hline & 3 & 1 & . & . & . & . & . \\
\hline & & 2 & . & . & . & . & . \\
\hline & 4 & 1 & . & . & . & . & . \\
\hline & & 2 & . & . & . & . & . \\
\hline \multirow[t]{8}{*}{ Uckermärker } & 1 & 1 & 5781 & 0 & 14,00 & 27 & 693 \\
\hline & & 2 & 4633 & 0 & 13,48 & 21 & 645 \\
\hline & 2 & 1 & 12327 & 0 & 65,50 & 18 & 807 \\
\hline & & 2 & 4262 & 0 & 65,00 & 23 & 820 \\
\hline & 3 & 1 & 343 & 0 & 55,70 & 22 & 670 \\
\hline & & 2 & 44 & 0 & 56,30 & 26 & 630 \\
\hline & 4 & 1 & 169 & 0 & 38,70 & 19 & 516 \\
\hline & & 2 & 18 & 0 & 8,20 & 25 & 241 \\
\hline
\end{tabular}

Geschl.=1: mănnl., Geschl.=2: wcibl., Geschl.=3: Mehrlinge weibl., Geschl.=4: Mehrlinge gemischt; Betrieb 1, Betrieb 2

\section{$2.2 \quad$ Methode}

Um die Parameter von Wachstumsfunktionen vernünftig schätzen zu können, müssen Beobachtungswerte von der Geburt bis zum erwachsenen Tier (Alter > 36 Monate) 
vorliegen (vgl. MATTHES et al., 1996). Die Beobachtungen in Tabelle 1 zeigen jedoch, daß dies nicht in allen Klassen der Fall ist. Deshalb ist auch zu erwarten, daß nicht für alle Klassifizierungen in Tabelle 1 eine geeignete Wachstumsfunktion bestimmbar ist. Unter diesen Gesichtspunkten wurde das Material reduziert, wobei auch auf die Unterscheidung nach Betrieben verzichtet wurde. Auf die Beschreibung des Wachstums gemischter Mehrlinge der Rasse Uckermärker wurde ebenfalls verzichtet, da die vorliegenden Daten nur einen einzigen Wert für ein Lebensalter $>36$ Monate enthalten. Die so gewonnene Neuklassifizierung, die die Basis für die Parameterschätzungen geeigneter Wachstumsfunktionen bildet, ist in Tabelle 2 dargestellt.

Tabelle 2

Neuklassifizicrung der Daten für die Parameterschätzungen (New classification of the data for parameter estimations)

\begin{tabular}{ccrcccc}
\hline Rasse & Geschl. & \multicolumn{1}{l}{ N } & $\min$. Alter & $\max$. Alter & min. LM & max. LM \\
\hline Charolais & 2 & 6559 & 0 & 74,50 & 22 & 834 \\
& 3 & 42 & 0 & 56,30 & 23 & 658 \\
Fleckvieh & 2 & 1169 & 0 & 95,50 & 25 & 760 \\
Uckermärker & 2 & 16589 & 0 & 65,50 & 18 & 820 \\
& 3 & 387 & 0 & 56,30 & 22 & 670 \\
\hline
\end{tabular}

Für die in der Tabelle 2 ausgewiesenen Klassifizierungen der Beobachtungswerte wurden mit der SAS-Prozedur NLIN Parameterschäzungen für fünf dreiparametrige Funktionen durchgeführt, die sich bereits zur Beschreibung des Wachstums von Rinderrassen als geeignet erwiesen haben (MATTHES et al., 1996). Zusätzlich wurden zwei vierparametrige Funktionen, die in der Literatur häufig verwendet werden (TAYLOR, 1977; SAGER, 1982; MATTHES et al., 1983; FRANCE und THORNLEY, 1984; MATTHES, 1985), in die Untersuchungen einbezogen. Dabei ist natürlich zu erwarten, daß die beiden vierparametrigen Funktionen kleinere Restvarianzschätzungen liefern werden als die dreiparametrigen Funktionen. Die sieben Wachstumsfunktionen sind in der Tabelle 3 zusammengestellt.

Tabelle 3

Verwendete Wachstumsfunktionen (Used growth functions)

\begin{tabular}{lcl}
\hline Name & Kurzbezeichnung & Formel \\
\hline Exponentialfunktion & Ex & $\mathrm{f}(\mathrm{x})=\mathrm{a}+\mathrm{be} \mathrm{e}^{\mathrm{cx}}$ \\
Gompertz-Funktion & Go & $\mathrm{f}(\mathrm{x})=\mathrm{a} \mathrm{e}^{\mathrm{be}}$ \\
Logistische Funktion & Lo & $\mathrm{f}(\mathrm{x})=\mathrm{a} /\left(1+\mathrm{be} \mathrm{e}^{\mathrm{cx}}\right.$ \\
Bertalanffy-Funktion & Be & $\left.\mathrm{f}(\mathrm{x})=(\mathrm{a}+\mathrm{be})^{\mathrm{cx}}\right)^{3}$ \\
tanh-Funktion & Th & $\mathrm{f}(\mathrm{x})=\mathrm{a} / 2(1+\tanh (\mathrm{c}(\mathrm{x}-\mathrm{b})))$ \\
Janoschek-Funktion & Ja & $\mathrm{f}(\mathrm{x})=\mathrm{a}+\mathrm{be} \mathrm{cx}^{\mathrm{d}}$ \\
Richards-Funktion & Ri & $\mathrm{f}(\mathrm{x})=\mathrm{a}\left(1+\mathrm{be} \mathrm{e}^{\mathrm{cx}}\right)^{\mathrm{d}}$ \\
\hline
\end{tabular}


Numerische Probleme bei der Schätzung der Parameter der Janoschek-Funktion und der Richards-Funktion mit der Prozedur NLIN für das Alter $x=0$ wurden mittels der Transformation $\mathrm{x}=\mathrm{x}+1$ gelöst.

Nach dem von MATTHES et al. (1996) vorgeschlagenen Optimalitätskriterium beschreibt diejenige Funktion innerhalb einer Klassifizierung nach Rasse und Geschlecht das Wachstum am besten, die die gewichtete Summe

$$
Q=a_{1}(G-\hat{G})^{2}+a_{2}(L-\hat{L})^{2}+a_{3} R V
$$

minimiert. G bzw. L bezeichnen in dieser Formel bekannte mittlere Werte für Geburts- und Lebendendmasse und $\hat{\mathrm{G}}$ bzw. $\hat{\mathrm{L}}$ sind die entsprechenden Werte der jeweiligen Wachstumsfunktion $\left(\hat{G}=f(0), \hat{L}=\lim _{x \rightarrow \infty} f(x)\right)$. Mit RV ist der Schätzwert der Restvarianz im entsprechenden nichtlinearen Regressionsmodell bezeichnet.

Abweichend vom obigen Optimalitätskriterium Q sollen hier die beiden folgenden Modifikationen verwendet werden:

$$
\begin{aligned}
& \mathrm{Q}_{1}=\mathrm{a}_{1}|\mathrm{G}-\hat{\mathrm{G}}|+\mathrm{a}_{2}\left|\mathrm{~L}_{\mathrm{m}}-\hat{\mathrm{L}}\right|+\mathrm{a}_{3} R V \text { und } \\
& \mathrm{Q}_{2}=\mathrm{a}_{1}|\mathrm{G}-\hat{\mathrm{G}}|+\mathrm{a}_{2}\left|\mathrm{~L}_{\max }-\hat{\mathrm{L}}\right|+\mathrm{a}_{3} R V \text {. }
\end{aligned}
$$

In beiden Kriterien wird als Vorinformation für $\mathrm{G}$ der (auf eine ganze Zahl gerundete) Mittelwert der beobachteten Geburtsmassen bezogen auf die jeweilige Klasse verwendet. $\mathrm{Q}_{1}$ und $\mathrm{Q}_{2}$ unterscheiden sich nur in der Verwendung unterschiedlicher Vorinformationen $\mathrm{L}_{m}$ und $\mathrm{L}_{\max }$ für die Lebendendmasse. In $\mathrm{Q}_{1}$ ist mit $\mathrm{L}_{\mathrm{m}}$ der (auf eine ganze Zahl gerundete) Mittelwert aller beobachteten Massen bezeichnet, die zu einem Alter > 36 Monate gehören. Dagegen ist $L_{\max }$ das Maximum dieser Beobachtungen.

Die Gewichte $a_{1}, a_{2}$ und $a_{3}$ werden aufgrund der folgenden drei Eigenschaften jeweils für jede Klasse bestimmt:

1. $a_{1}+a_{2}+a_{3}=1$

2. $\mathrm{a}_{1} / \mathrm{a}_{2} \approx \mathrm{L} / \mathrm{G}$

3. $a_{3} \prec a_{1}+a_{2}$.Die Werte $G, L_{m}, L_{\max }$ und die daraus bestimmten Quotienten sind in der Tabelle 4 zusammengestellt.

Werte für $G, L_{m}$ und $L_{\max }$ (Values for $G, L_{m}$ und $L_{m a x}$ )

\begin{tabular}{lcccccc}
\hline RASSE & $\mathrm{Ges}$ & $\mathrm{G}$ & $\mathrm{L}_{\mathrm{m}}$ & $\mathrm{L}_{\max }$ & $\mathrm{L}_{\mathrm{m}} / \mathrm{G}$ & $\mathrm{L}_{\max } / \mathrm{G}$ \\
\hline Charolais & 2 & 42 & 631 & 834 & 15,02 & 19,86 \\
Charolais & 3 & 32 & 601 & 658 & 18,78 & 20,56 \\
Fleckvieh & 2 & 38 & 527 & 760 & 13,87 & 20,00 \\
Uckermärker & 2 & 41 & 603 & 820 & 14,71 & 20,00 \\
Uckermärker & 3 & 33 & 583 & 670 & 17,67 & 20,30 \\
\hline
\end{tabular}


Zur Bestimmung der Gewichte $a_{1}, a_{2}$ und $a_{3}$ wird für $Q_{1} L_{m} / G \approx 15=a_{1} / a_{2}$ und für $Q_{2}$ $L_{\max } / G \approx 20=a_{1} / a_{2}$ gesetzt. $a_{3}$ wird nach der o. g. Eigenschaft 3 klein gegen $a_{1}+a_{2}$ gewählt derart, daß der Anteil $a_{3} R V$ in $Q$ nicht zu groß ist gegenüber der Summe $a_{1}|G-\hat{G}|+a_{2}|L-\hat{L}|$. Wie die Parameterschätzungen zeigen, liegen die Restvarianzen in der Größenordnung $10^{3}$. Deshalb wählen wir $\mathrm{a}_{3}=0.001$. Daraus ergeben sich folgenden Gewichte:

Tabelle 5

Gewichte $a_{1}, a_{2}$ und $a_{3}$ (Weights $a_{1}, a_{2}$ und $a_{3}$ )

\begin{tabular}{cccc}
\hline $\mathrm{Q}$ & $\mathrm{a}_{1}$ & $\mathrm{a}_{2}$ & $\mathrm{a}_{3}$ \\
\hline $\mathrm{Q}_{1}$ & 0,9366 & 0,0624 & 0,0010 \\
$\mathrm{Q}_{2}$ & 0,9514 & 0,0476 & 0,0010 \\
\hline
\end{tabular}

Neben der Restvarianz und den beiden Funktionen $\mathrm{Q}_{1}$ und $\mathrm{Q}_{2}$ können auch weitere Anpassungskriterien zur Bestimmung einer "besten“" Wachstumsfunktion verwendet werden. Die Anpassungskriterien von AKAIKE und SCHWARTZ (RASCH, 1995) berücksichtigen insbesondere die Anzahl der Parameter der jeweiligen Funktion. Diese beiden Kriterien sollen, da hier sowohl drei- als auch vierparametrige Wachstumsfunktionen verwendet werden, ebenfalls berechnet werden.

Wenn mit N und RV die Anzahl der Beobachtungen und die geschätzte Restvarianz in der jeweiligen Klasse sowie mit $p$ die Anzahl der Parameter der jeweiligen Wachstumsfunktion bezeichnet werden, dann berechnen sich die beiden Kriterien wie folgt:

$$
\begin{array}{ll}
\text { Akaike-Kriterium: } & \mathrm{AIC}=\mathrm{N} \ln \left(\frac{\mathrm{N}-\mathrm{p}}{2} \mathrm{RV}\right)+\frac{\mathrm{N}(\mathrm{n}+\mathrm{p})}{\mathrm{N}-\mathrm{p}-2}, \\
\text { Schwartz-Kriterium: } & \mathrm{SC}=\mathrm{N} \ln \left(\frac{\mathrm{N}-\mathrm{p}}{\mathrm{N}} \mathrm{RV}\right)+\mathrm{p} \ln (\mathrm{N}) .
\end{array}
$$

3. Ergebnisse und Diskussion

Die Tabelle 6 enthält für die Klassifizierungen nach Tabelle 2 die geschätzten Parameterwerte, die zugehörige Restvarianz und die aus den Schätzwerten für die Paramater $\mathrm{a}, \mathrm{b}, \mathrm{c}$ und $\mathrm{d}$ berechneten maximalen Lebendmassen $(\hat{\mathrm{L}})$ und die den geschätzten Geburtsmassen $(\hat{G})$ entsprechenden Werte $f(0)$ (bzw. $f(1)$ im Falle der JanoschekFunktion und der Richards-Funktion). Die Ergebnisse in Tabelle 6 sind innerhalb von Rasse und Geschlecht nach wachsender Restvarianz sortiert.

Wie zu erwarten war, liefern die beiden vierparametrigen Wachstumsfunktionen gegenüber allen dreiparametrigen Funktionen eine bessere Anpassung im Sinne einer kleineren Restvarianz. Die Unterschiede zwischen den Restvarianzschätzungen für die beiden vierparametrigen Funktionen sind äußerst gering. Mit ebenfalls geringem Unterschied in der Restvarianzschätzung zu den beiden vierparametrigen Wachstumsfunktionen liefert in allen fünf Klassen die dreiparametrige Exponentialfunktion die 
beste Anpassung im Hinblick auf die Restvarianz.

Tabelle 6

Parameterschätzungen und berechnete Größen (Parameter estimations and computed values)

\begin{tabular}{|c|c|c|c|c|c|c|c|c|c|c|}
\hline Rasse & Ges. & $\mathbf{N}$ & a & b & c & d & $\hat{\mathbf{G}}$ & $\hat{\mathbf{L}}$ & RV & Fkt. \\
\hline \multirow[t]{7}{*}{ Charolais } & 2 & 6559 & 709.40 & -710.57 & -0.06 & 0.93 & 41.50 & 709.40 & 1599.84 & $\mathrm{Ja}$ \\
\hline & & & 702.30 & -1.00 & -0.05 & 0.89 & 41.64 & 702.30 & 1601.14 & $\mathrm{Ri}$ \\
\hline & & & 686.23 & -642.99 & -0.05 & & 43.24 & 686.23 & 1607.77 & Ex \\
\hline & & & 8.59 & -4.72 & -0.09 & & 57.96 & 634.48 & 1864.36 & $\mathrm{Be}$ \\
\hline & & & 621.25 & -2.25 & -0.11 & & 65.77 & 621.25 & 2071.24 & Go \\
\hline & & & 598.93 & 6.26 & -0.17 & & 82.48 & 598.93 & 2693.67 & Lo \\
\hline & & & 599.02 & 10.63 & 0.09 & & 82.63 & 599.02 & 2693.89 & Th \\
\hline \multirow[t]{7}{*}{ Charolais } & 3 & 42 & 649.91 & -0.95 & -0.07 & 1.37 & 32.01 & 649.91 & 720.92 & $\mathrm{Ri}$ \\
\hline & & & 644.43 & -635.09 & -0.04 & 1.16 & 31.82 & 644.43 & 724.11 & $\mathrm{Ja}$ \\
\hline & & & 688.83 & -659.44 & -0.05 & & 29.39 & 688.83 & 741.33 & Ex \\
\hline & & & 8.50 & -5.12 & -0.10 & & 38.93 & 615.19 & 777.09 & $\mathrm{Be}$ \\
\hline & & & 598.04 & -2.58 & -0.12 & & 45.52 & 598.04 & 920.03 & Go \\
\hline & & & 574.01 & 10.57 & 0.10 & & 60.64 & 574.01 & 1428.98 & Th \\
\hline & & & 574.04 & 8.47 & -0.20 & & 60.65 & 574.04 & 1428.98 & Lo \\
\hline \multirow[t]{7}{*}{ Fleckvieh } & 2 & 1169 & 563.16 & -0.94 & -0.07 & 1.32 & 33.04 & 563.16 & 2136.52 & $\mathrm{Ri}$ \\
\hline & & & 561.34 & -550.61 & -0.04 & 1.12 & 31.85 & 561.34 & 2146.13 & $\mathrm{Ja}$ \\
\hline & & & 579.32 & -553.96 & -0.05 & & 25.36 & 579.32 & 2162.06 & Ex \\
\hline & & & 8.17 & -4.60 & -0.09 & & 45.54 & 544.75 & 2174.10 & $\mathrm{Be}$ \\
\hline & & & 536.21 & -2.30 & -0.11 & & 53.53 & 536.21 & 2241.56 & Go \\
\hline & & & 522.35 & 10.97 & 0.09 & & 68.54 & 522.35 & 2468.54 & $\mathrm{Th}$ \\
\hline & & & 522.29 & 6.63 & -0.17 & & 68.46 & 522.29 & 2468.54 & Lo \\
\hline \multirow[t]{7}{*}{ Uckermärker } & 2 & 16589 & 667.85 & -667.13 & -0.06 & 0.96 & 40.89 & 667.85 & 1416.20 & $\mathrm{Ja}$ \\
\hline & & & 662.93 & -1.00 & -0.05 & 0.94 & 41.10 & 662.93 & 1417.00 & $\mathrm{Ri}$ \\
\hline & & & 654.66 & -612.60 & -0.06 & & 42.07 & 654.66 & 1418.52 & Ex \\
\hline & & & 8.45 & -4.59 & -0.10 & & 57.54 & 603.04 & 1595.08 & $\mathrm{Be}$ \\
\hline & & & 589.79 & -2.19 & -0.12 & & 65.69 & 589.79 & 1746.96 & Go \\
\hline & & & 567.22 & 9.83 & 0.09 & & 83.66 & 567.22 & 2220.73 & Th \\
\hline & & & 567.18 & 5.78 & -0.18 & & 83.66 & 567.18 & 2220.73 & Lo \\
\hline \multirow[t]{7}{*}{ Uckermärker } & 3 & 387 & 611.66 & -0.95 & -0.07 & 1.33 & 32.55 & 611.66 & 752.25 & $\mathrm{Ri}$ \\
\hline & & & 608.13 & -598.86 & -0.04 & 1.14 & 32.25 & 608.13 & 756.40 & $\mathrm{Ja}$ \\
\hline & & & 645.77 & -616.85 & -0.05 & & 28.92 & 645.77 & 777.38 & Ex \\
\hline & & & 8.33 & -4.86 & -0.10 & & 41.84 & 577.33 & 810.78 & $\mathrm{Be}$ \\
\hline & & & 560.58 & -2.43 & -0.12 & & 49.37 & 560.58 & 915.87 & Go \\
\hline & & & 532.74 & 9.95 & 0.10 & & 65.89 & 532.74 & 1295.05 & $\mathrm{Th}$ \\
\hline & & & 532.73 & 7.09 & -0.20 & & 65.89 & 532.73 & 1295.05 & Lo \\
\hline
\end{tabular}

Aus der folgenden Tabelle 7, in der die Werte für die Optimalitätskriterien $Q_{1}$ und $Q_{2}$ (auf zwei Dezimalstellen gerundet) zusammengestellt sind, ist ersichtlich, ob bei Berücksichtigung der beiden Optimalitätskriterien andere Wachstumsfunktionen favorisiert werden. Eine durch das Optimalitätskriterium $\mathrm{Q}_{1}$ oder $\mathrm{Q}_{2}$ bedingte Verbesserung in der Reihenfolge der Funktionen, die innerhalb von Rasse und Geschlecht wie in Tabelle 6 nach wachsender Restvarianz geordnet sind, ist jeweils mit einem Stern gekennzeichnet. 
Tabelle 7

Werte der Funktionen $Q_{1}$ und $Q_{2}$ (Values of the functions $Q_{1}$ and $Q_{2}$ )

\begin{tabular}{|c|c|c|c|c|c|c|c|c|c|}
\hline RASSE & GES & Funktion & RV & & $\mathbf{Q}_{1}$ & $\mathbf{Q}_{2}$ & $\mathbf{G}-\hat{\mathbf{G}}$ & $\mathbf{L}_{m}-\hat{\mathbf{L}}$ & $\mathbf{L}_{\max }-\hat{\mathbf{L}}$ \\
\hline \multirow[t]{7}{*}{ Charolais } & 2 & $\mathrm{Ja}$ & 1599.84 & & 7.04 & 8.09 & 0.59 & -78.40 & 124.60 \\
\hline & & $\mathrm{Ri}$ & 1601.14 & & 6.47 & 8.30 & 0.45 & -71.30 & 131.70 \\
\hline & & Ex & 1607.77 & " & 6.13 & 9.74 & -1.15 & -55.23 & 147.77 \\
\hline & & $\mathrm{Be}$ & 1864.36 & & 16.95 & 26.46 & -15.87 & -3.48 & 199.52 \\
\hline & & Go & 2071.24 & & 24.86 & 34.73 & -23.68 & 9.75 & 212.75 \\
\hline & & Lo & 2693.67 & & 42.52 & 52.31 & -40.39 & 32.07 & 235.07 \\
\hline & & Th & 2693.89 & & 42.66 & 52.45 & -40.54 & 31.98 & 234.98 \\
\hline \multirow[t]{7}{*}{ Charolais } & 3 & $\mathrm{Ri}$ & 720.92 & & 3.85 & 1.18 & -0.08 & -48.91 & 8.09 \\
\hline & & $\mathrm{Ja}$ & 724.11 & * & 3.54 & 1.47 & 0.11 & -43.43 & 13.57 \\
\hline & & Ex & 741.33 & & 8.60 & 4.63 & 2.54 & -87.83 & -30.83 \\
\hline & & $\mathrm{Be}$ & 777.09 & * & 8.22 & 9.47 & -7.00 & -14.19 & 42.81 \\
\hline & & Go & 920.03 & & 13.83 & 16.70 & -13.59 & 2.96 & 59.96 \\
\hline & & Th & 1428.98 & & 30.00 & 32.74 & -28.71 & 26.99 & 83.99 \\
\hline & & Lo & 1428.98 & & 30.01 & 32.75 & -28.72 & 26.96 & 83.96 \\
\hline \multirow[t]{7}{*}{ Fleckvich } & 2 & $\mathrm{Ri}$ & 2136.52 & & 8.62 & 15.80 & 4.51 & -36.16 & 196.84 \\
\hline & & $\mathrm{Ja}$ & 2146.13 & & 9.63 & 17.03 & 5.70 & -34.34 & 198.66 \\
\hline & & Ex & 2162.06 & & 16.84 & 22.36 & 12.19 & -52.32 & 180.68 \\
\hline & & $\mathrm{Be}$ & 2174.10 & * & 10.77 & * 20.02 & -7.99 & -17.75 & 215.25 \\
\hline & & Go & 2241.56 & & 17.78 & 28.10 & -15.98 & -9.21 & 223.79 \\
\hline & & Th & 2468.54 & & 31.78 & 43.26 & -30.99 & 4.65 & 237.65 \\
\hline & & Lo & 2468.54 & * & 31.71 & 43.19 & -30.91 & 4.71 & 237.71 \\
\hline \multirow[t]{7}{*}{ Uckermärker } & 2 & $\mathrm{Ja}$ & 1416.20 & & 5.95 & 9.15 & 0.52 & -64.85 & 152.15 \\
\hline & & $\mathrm{Ri}$ & 1417.00 & * & 5.45 & 9.19 & 0.31 & -59.93 & 157.07 \\
\hline & & Ex & 1418.52 & $*$ & 5.26 & 9.92 & -0.66 & -51.66 & 165.34 \\
\hline & & $\mathrm{Be}$ & 1595.08 & & 16.70 & 27.27 & -16.13 & -0.04 & 216.96 \\
\hline & & Go & 1746.96 & & 25.31 & 35.80 & -24.28 & 13.21 & 230.21 \\
\hline & & Th & 2220.73 & & 44.02 & 54.45 & -42.25 & 35.78 & 252.78 \\
\hline & & Lo & 2220.73 & & 44.03 & 54.45 & -42.25 & 35.82 & 252.82 \\
\hline \multirow[t]{7}{*}{ Uckermärker } & 3 & $\mathrm{Ri}$ & 752.25 & & 3.11 & 4.11 & 0.61 & -28.66 & 58.34 \\
\hline & & $\mathrm{Ja}$ & 756.40 & & 3.18 & 4.57 & 0.91 & -25.13 & 61.87 \\
\hline & & Ex & 777.38 & & 8.67 & 5.96 & 4.24 & -62.77 & 24.23 \\
\hline & & $\mathrm{Be}$ & 810.78 & & 9.29 & 13.48 & -8.68 & 5.67 & 92.67 \\
\hline & & Go & 915.87 & & 17.50 & 21.55 & -16.21 & 22.42 & 109.42 \\
\hline & & Th & 1295.05 & & 35.09 & 38.97 & -32.73 & 50.26 & 137.26 \\
\hline & & Lo & 1295.05 & & 35.09 & 38.97 & -32.73 & 50.27 & 137.27 \\
\hline
\end{tabular}

In der Tabelle 8 sind die Werte für alle Anpassungskriterien zusammengefaßt. Die Funktionen sind innerhalb von Rasse und Geschlecht wieder nach wachsender Restvarianz geordnet. In den Spalten für die Anpassungskriterien $\mathrm{Q}_{1}, \mathrm{Q}_{2}, \mathrm{AIC}$ und SC sind die jeweils drei kleinsten Kriteriumswerte mit nachstehenden (1), (2), (3) gekennzeichnet, falls sich Veränderungen gegenüber dem Restvarianzkriterium ergeben.

In den Klassen weiblicher Charolais- und Uckermärker Rinder liefern die Janoschek-, Richards- und die dreiparametrige Exponentialfunktion die besten Anpassungen sowỏhl nach dem Restvarianz-, Akaike-, Schwartz- und $\mathrm{Q}_{2}$-Kriterium bei geringen Unterschieden zwischen den Kriteriumswerten für die drei Funktionen. Das $Q_{1}-$ Kriterium 
kehrt diese Reihenfolge um und favorisiert die Exponentialfunktion.

Tabelle 8

Werte der Kriterien $\mathrm{Q}_{1}, \mathrm{Q}_{2}, \mathrm{AIC}$ und SC (Values of the functions $\mathrm{Q}_{1}, \mathrm{Q}_{2}$, AIC und SC)

\begin{tabular}{|c|c|c|c|c|c|c|c|c|c|c|}
\hline RASSE & GES & Funktion & RV & $\mathbf{Q}_{1}$ & & $\mathbf{Q}_{2}$ & & AIC & SC & \\
\hline \multirow[t]{7}{*}{ Charolais } & 2 & $\mathrm{Ja}$ & 1599.84 & 7.04 & (3) & 8.09 & & 108053.10 & 48421.22 & \\
\hline & & $\mathrm{Ri}$ & 1601.14 & 6.47 & (2) & 8.30 & & 108058.43 & 48426.55 & \\
\hline & & Ex & 1607.77 & 6.13 & (1) & 9.74 & & 108084.53 & 48445.86 & \\
\hline & & $\mathrm{Be}$ & 1864.36 & 16.95 & & 26.46 & & 109055.72 & 49417.05 & \\
\hline & & Go & 2071.24 & 24.86 & & 34.73 & & 109745.92 & 50107.25 & \\
\hline & & Lo & 2693.67 & 42.52 & & 52.31 & & 111469.35 & 51830.68 & \\
\hline & & Th & 2693.89 & 42.66 & & 52.45 & & 111469.88 & 51831.21 & \\
\hline \multirow[t]{7}{*}{ Charolais } & 3 & $\mathrm{Ri}$ & 720.92 & 3.85 & (2) & 1.18 & & $453.72 \quad(2)$ & 287.13 & (2) \\
\hline & & $\mathrm{Ja}$ & 724.11 & 3.54 & (1) & 1.47 & & 453.90 & 287.31 & (3) \\
\hline & & Ex & 741.33 & 8.60 & & 4.63 & & 453.39 (1) & 285.66 & (1) \\
\hline & & $\mathrm{Be}$ & 777.09 & 8.22 & (3) & 9.47 & & 455.37 & 287.63 & \\
\hline & & Go & 920.03 & 13.83 & & 16.70 & & 462.46 & 294.73 & \\
\hline & & Th & 1428.98 & 30.00 & & 32.74 & & 480.96 & 313.22 & \\
\hline & & Lo & 1428.98 & 30.01 & & 32.75 & & 480.96 & 313.22 & \\
\hline \multirow[t]{7}{*}{ Fleckvieh } & 2 & $\mathrm{Ri}$ & 2136.52 & 8.62 & (1) & 15.80 & (1) & 17585.10 & 8986.89 & \\
\hline & & $\mathrm{Ja}$ & 2146.13 & 9.63 & (2) & 17.03 & (2) & 17590.35 & 8992.14 & \\
\hline & & Ex & 2162.06 & 16.84 & & 22.36 & & 17597.98 & 8994.72 & \\
\hline & & $\mathrm{Be}$ & 2174.10 & 10.77 & (3) & 20.02 & (3) & 17604.47 & 9001.22 & \\
\hline & & Go & 2241.56 & 17.78 & & 28.10 & & 17640.20 & 9036.94 & \\
\hline & & Th & 2468.54 & 31.78 & & 43.26 & & 17752.95 & 9149.69 & \\
\hline & & Lo & 2468.54 & 31.71 & & 43.19 & & 17752.95 & 9149.69 & \\
\hline \multirow[t]{7}{*}{ Uckermärker } & 2 & $\mathrm{Ja}$ & 1416.20 & 5.95 & (3) & 9.15 & & 286648.67 & 120400.21 & \\
\hline & & $\mathrm{Ri}$ & 1417.00 & 5.45 & (2) & 9.19 & & 286658.04 & 120409.58 & \\
\hline & & Ex & 1418.52 & 5.26 & (1) & 9.92 & & 286674.82 & 120418.65 & \\
\hline & & $\mathrm{Be}$ & 1595.08 & 16.70 & & 27.27 & & 288620.87 & 122364.70 & \\
\hline & & Go & 1746.96 & 25.31 & & 35.80 & & 290129.70 & 123873.53 & \\
\hline & & Th & 2220.73 & 44.02 & & 54.45 & & 294110.37 & 127854.20 & \\
\hline & & Lo & 2220.73 & 44.03 & & 54.45 & & 294110.37 & 127854.20 & \\
\hline \multirow[t]{7}{*}{ Uckermärker } & 3 & $\mathrm{Ri}$ & 752.25 & 3.11 & & 4.11 & & 4993.93 & 2582.94 & \\
\hline & & $\mathrm{Ja}$ & 756.40 & 3.18 & & 4.57 & & 4996.06 & 2585.07 & \\
\hline & & Ex & 777.38 & 8.67 & & 5.96 & & 5005.60 & 2590.71 & \\
\hline & & $\mathrm{Be}$ & 810.78 & 9.29 & & 13.48 & & 5021.88 & 2606.99 & \\
\hline & & Go & 915.87 & 17.50 & & 21.55 & & 5069.05 & 2654.15 & \\
\hline & & Th & 1295.05 & 35.09 & & 38.97 & & 5203.12 & 2788.22 & \\
\hline & & Lo & 1295.05 & 35.09 & & 38.97 & & 5203.12 & 2788.22 & \\
\hline
\end{tabular}

Bei den weiblichen Rinder der Rasse Fleckvieh haben die Richards- und die Janoschekfunktion die kleinsten Werte für alle fünf Kriterien. Die drittbeste Anpassung liefert die Exponentialfunktion für das Restvarianz-, Akaike- und Schwartzkriterium und die Bertalanffy-Funktion für die Kriterien $\mathrm{Q}_{1}$ und $\mathrm{Q}_{2}$.

Während bei den weiblichen Mehrlingen der Rasse Uckermärker alle fünf Kriterien die Richards-, Janoschek- und Exponentialfunktion als die drei am besten angepaßten Funktionen auswählen, liefern bei den weiblichen Mehrlingen der Rasse Charolais nur das Restvarianz und das $\mathrm{Q}_{2}$-Kriterium die gleiche Auswahl. 
In der folgenden Abbildung 1 sind für die drei Rassen weiblicher Rinder die jeweils im Sinne des Optimalitätskriteriums $\mathrm{Q}_{2}$ am besten angepaßten Funktionen dargestellt.

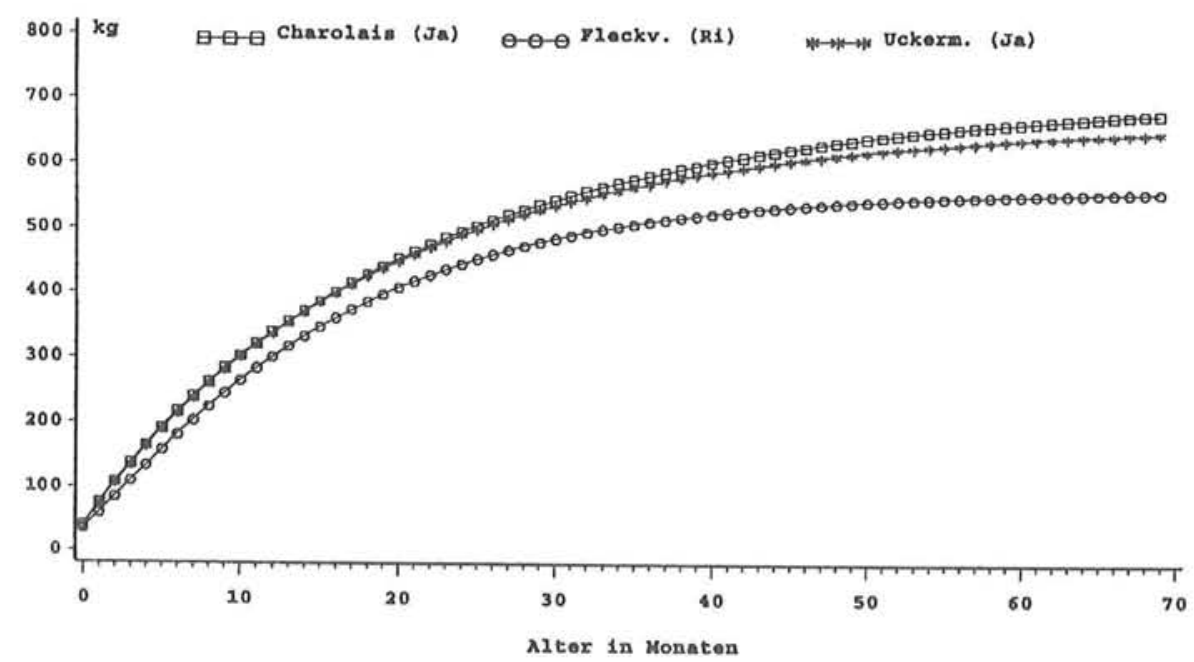

Abb. 1: Wachstum weiblicher Rinder gemäß der besten Funktion bzgl. $Q_{2}$ (Growth of female cows in accordance with the best function with respect to $Q_{2}$ )

Die Abbildung 2 zeigt die unter Verwendung der in Abbildung 1 dargestellten Funktionen berechneten mittleren monatlichen Zuwächse für die weiblichen Rinder der Rassen Charolais, Fleckvieh und Uckermärker.

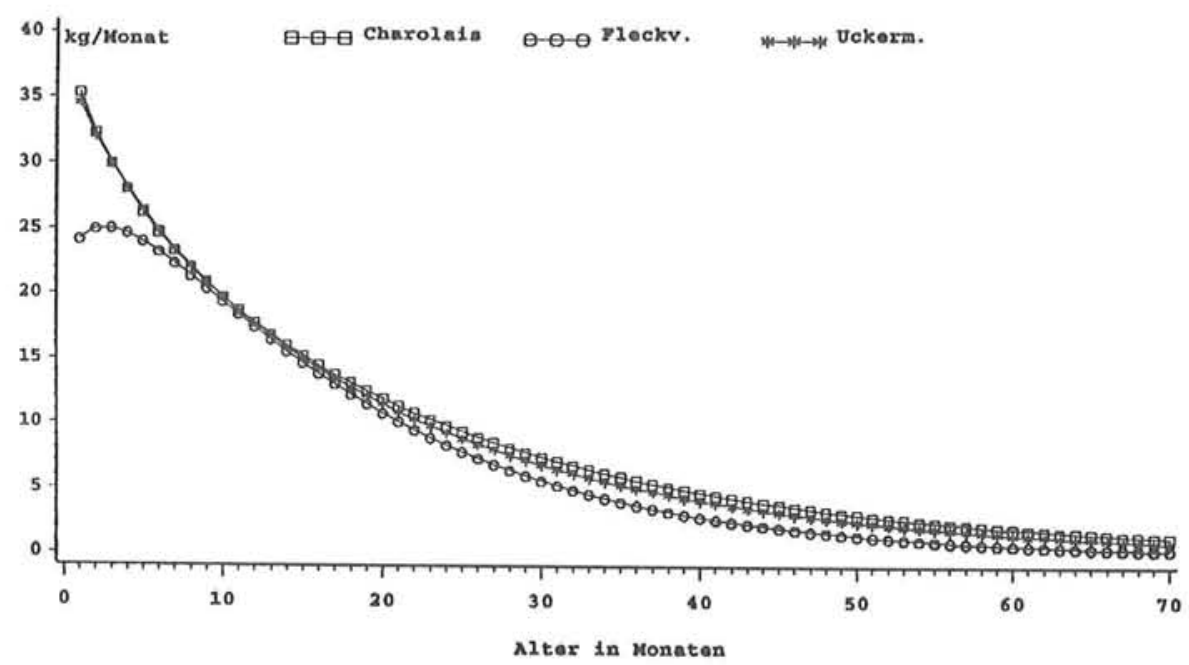

Abb. 2: Monatliche Zunahmen weiblicher Rinder (Monthly increases of female cows) 
Nur für die Rasse Fleckvieh ist aus Abbildung 2 ersichtlich, daß die maximale Lebendmassezunahme nicht im ersten Lebensmonat erfolgt. Die untypischen Verläufe mit einer maximalen Gewichtszunahme im ersten Lebensmonat für die Rassen Charolais bzw. Uckermärker sind wahrscheinlich auf die sehr große Anzahl von Beobachtungen (6559 bzw. 16589) zurückzuführen.

Die Abbildungen 3 und 4 zeigen das Wachstum und die mittleren monatlichen Zunahmen für weibliche Mehrlinge der Rassen Charolais und Uckermärker wieder unter Verwendung der im Sinne von Optimalitätskriterium $\mathrm{Q}_{2}$ am besten angepaßten Funktionen

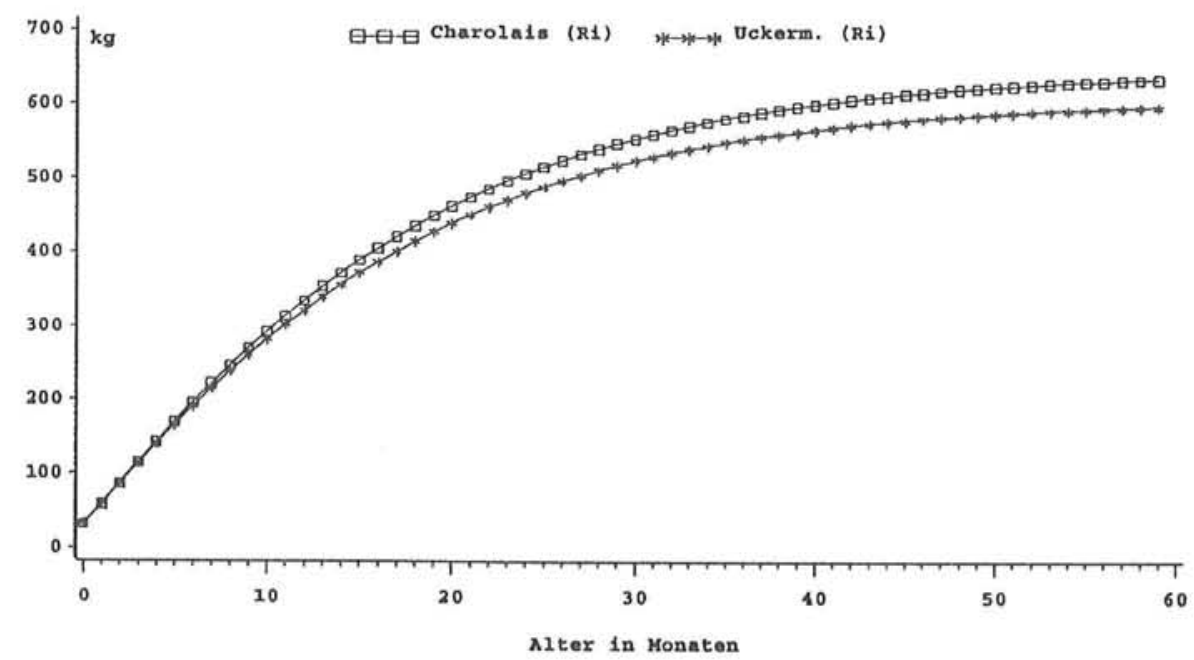

Abb. 3: Wachstum weiblicher Mehrlinge gemäß der besten Funktion bzgl. $Q_{2}$ (Growth of female twins in accordance with the best function with respect to $Q_{2}$ )

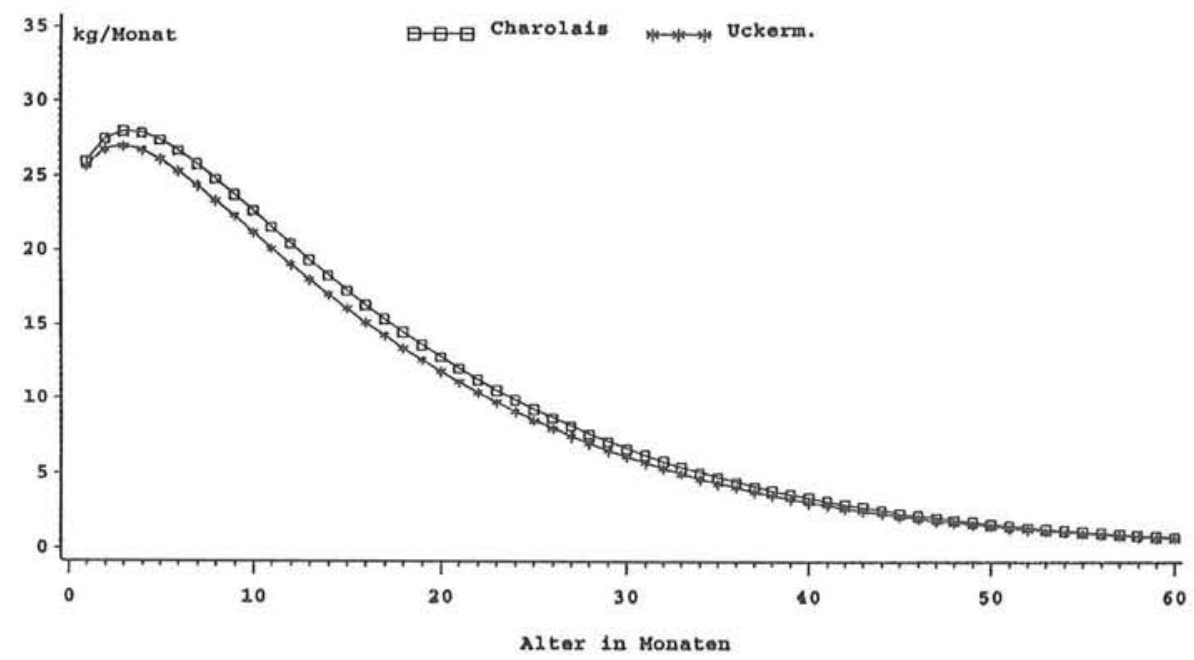

Abb. 4. Monatliche Zunahmen weiblicher Mehrlinge (Monthly increases of female twins) 


\section{Literatur}

BAKKER, U.; KOOPS, W.:

An Approach to the Comparison of Growth Curves of Dutch Friesian, British Friesian and Holstein Friesian cows. Pattern of Growth and Development in Cattle. Seminar in the EEC Programme of Coordination of Research on Beef Production held at Chent, 11. - 13.10.1977, 2 - 11

FRANCE, J.; THORNLEY, J. H. M.:

Growth Functions as Mathematical Models in Agriculture. Butterworth London, Boston, 1984

MATTHES, H.-D.:

Beitrag zur züchterischen Verbesserung der Fleischleistung in der Milchrindpopulation. Univ. Rostock, Diss. B, 1985

MATTHES, H.-D.; RUDOLPH, P. E.; HERRENDÖRFER, G.:

Verwendung von Wachstumsfunktionen zur Charakterisierung des Wachstums von Fleischrindrassen. Arch. Tierz., Dummerstorf 39 (1996), 121-127

MATTHES, H.-D.; SAGER, G.; PANICKE, L.; PETERS, H.:

Charakterisierung des Wachstumsverlaufes über Wachstumsfunktionen bei männlichen und weiblichen RASCH, D: Jungrindern. Arch. Tierz., Berlin 26 (1983), 499-510

Einführung in die mathematische Beschreibung des Wachstums einschließlich Literaturüberblick. Probleme der angewandten Statistik, (1984) 11, 5-29

RASCH, D.; GUIARD, V.; NƯRNBERG, G.:

Statistische Versuchsplanung. Einführung in die Methoden und Anwendung des Dialogsystems SAGER, G.: CADEMO. Gustav Fischer Verlag, Stuttgart · Jena · New York 1992, 386 S.

Mathematische Formulierungen des Höhenwachstums von Holstein-Rindern. Arch. Tierz., Berlin 25 SAGER, G.: (1982), 185-194

Zur Erfassung nahrungsbedingter Modifikation bei Wachstumsabläufen. Zool. Jahrb. Anat., 109 (1983), $451-465$

SAS LANGUAGE:

Reference, Version 6, First Edition,Cary, NC: SAS Institute Inc., 1990

SAS/STAT Users Guide):

Version 6, Forth Edition, Volume 2, Cary,NC: SAS Institute Inc., 1989

TAYLOR, C. S.:

Methods of Quantifying Growth and Development. General Review. Pattern of Growth and Development in Cattle. A Seminar in the EEC Programme of Coordination of Research on Beef Production held at Gent, October 11-13, 1977

TONG, A.K.W.:

Breed Averages and Age of Dam Adjustment Factors for Birth Weight of Beef Cattle. Can. J. Anim. Sci., Ottawa 63 (1983), 7-15

Eingegangen: 22.04.1998

Akzeptiert: 15.11 .1998

Anschrif der Verfasser

Prof. Dr. agr. habil. HEIDE-DÖRTE MATTHES

Dr. PAUL EBERHARD RUDOLPH

Forschungsinstitut für die Biologie landwirtschaftlicher Nutztiere (FBN)

Wilhelm-Stahl-Allee 2

D-18196 Dummerstorf 
Arch. Tierz., Dummerstorf 42 (1999) 4, 346

Buchbesprechung

\author{
Fertilitätsstörungen beim weiblichen Rind \\ EBERHARD GRUNERT und AART DE KRUIF
}

3. neubearbeitete Auflage, Parey Buchverlag Berlin 1999, 430 Seiten, 446 Abbildungen, davon 130 auf Farbtafeln, 49 Tabellen, ISBN 3-8263-3150-8, 248,00 DM

Die Monographie Fertilitätsstörungen beim weiblichen Rind zählt seit ihrer ersten Auflage zu den deutschsprachigen Standardwerken auf dem Fachgebiet. Unter Mitarbeit nationaler und internationalen Autoren haben die beiden Herausgeber Eberhard Grunert und Aart de Kruif in der nunmehr dritten Auflage des Lehrbuches das aktuelle Wissen auf dem Gebiet in 21 Kapitel strukturiert. Es erscheint bereits einleitend bemerkenswert, daß es den Herausgebern gelungen ist, den Umfang des Lehrbuches im Vergleich zur zweiten Auflage zu reduzieren, obwohl zahlreiche neue Erkenntnisse in die einzelnen Abschnitte eingearbeitet wurden. Die Vereinigung beider Zielstellungen wurde nicht nur durch einen Verzicht auf die in der ersten und zweiten Auflage vorkommenden Kapitel „Bedeutung der Fruchtbarkeitsstörungen“ und „Embryologie und Anatomie des weiblichen Genitale" sondern vor allem durch eine konsequente Überarbeitung des gesamten Lehrbuches ermöglicht. Als Beispiele für die Integration von neuen Erkenntnissen in die dritte Auflage seien die Ausführungen zur Anwendung der bildgebenden Ultraschalldiagnostik im Rahmen der Trächtigkeitsdiagnostik oder die Informationen über neuere Reproduktionstechniken genannt. Den Autoren der einzelnen Abschnitte ist es in hervorzuhebender Weise gelungen, dem Leserkreis die wesentlichsten Informationen zu den verschiedenen Teilthemen in kurzer, übersichtlicher und einprägsamer Form zu vermitteln. Einen wesentlichen Beitrag dazu leisten die informativen Schemata, die zahlreichen aussagefähigen und häufig auch farbigen Abbildungen sowie die übersichtlichen Tabellen Das prinzipielle didaktische Konzept des Lehrbuches, welches von physiologischen Grundlagen ausgehend über eine fundierte Diagnostik die gezielte und effektive Therapie bzw. Prophylaxe von Fortpflanzungsstörungen zum Ziel hat, wurde sowohl durch die Gliederung der Thematik unter inhaltlichen Aspekten als auch durch eine ausgewogene Verbindung von Text und anderen gestalterischen Elementen sehr gut umgesetzt. In diesem Zusammenhang wird auf das Bestreben der Autoren verwiesen, den Lesern bei allen Bemühungen um inhaltliche Aktualität der einzelnen Kapitel gesicherte Erkenntnisse zu vermitteln, die einen praktische Anwendung dieser gestatten. Gerade in der Verbindung von Wissensvermittlung und der Nutzung von Erkenntnissen in der tierärztlichen Praxis liegt der besondere Wert des Lehrbuches, welches dem interessierten Leserkreis in seiner neuesten Auflage bestens empfohlen werden kann.

WILHELM KANITZ, Dummerstorf 